\title{
Analysis of Microsatellite Instability in Colorectal Cancer and Precursor Lesions
}

\author{
Ge Ye, Hong Chen, Jinku Zhang*, Jirui Sun* \\ Department of Pathology, the First Central Hospital of Baoding City, Hebei Key Laboratory of Molecular Pathology and \\ Early Diagnosis of Tumor, Baoding City, Hebei Province, China \\ *Corresponding authors: Jinku Zhang, zjkblk@sina.com; Jirui Sun, 75111863@qq.com

\begin{abstract}
In many countries, colorectal cancer is one of the common cancers. It has been confirmed that microsatellite colorectal cancer, adenomas and some polyps also have MSI phenomenon. This article analyzes the MSI of colorectal cancer and its closely related adenomas and polyps in domestic and foreign literature, and further discusses how to identify colorectal cancer at an early stage and the impact of MSI on the prognosis and treatment of colorectal cancer.
\end{abstract} \\ instability (MSI) is one of the most important molecular changes in colorectal cancer. As the recognized precursor lesions of
}

Keywords: Colorectal cancer; Adenomas; Polyps; Microsatellite instability

Publication date: July 2021; Online publication: July 30, 2021

\section{Introduction}

In 2020, the incidence rate of colorectal cancer ranked third and mortality rate ranked second in 19.3 million of the new cancer cases in the world ${ }^{[1]}$. According to the latest data collected by the National Cancer Institute (NCI)'s Surveillance, Epidemiology and End Results (SEER) program, in 2020, the number of newly diagnosed colorectal cancer in the United States will reach more than 100,000, including 104,610 cases of colon cancer and 43,340 cases of rectal cancer, and the death toll is expected to reach more than 50,000. Although most of the cases occur in people over 50 years old, 17,930 new cases (12\%) will be diagnosed in people under 50 years old ${ }^{[2]}$; in Australia, the United Kingdom, Germany and many other countries, the number of patients under the age of 50 is also on the rise in varying degrees ${ }^{[3]}$. This shows that colorectal cancer is not only a threat to middle-aged people, its risk is also apparent in the younger generations. Although the genetic mechanism of colorectal cancer is not very clear, it has been found that adenoma is a recognized precursor lesion (adenoma cancer sequence) in colorectal cancer. This genetic model was first proposed by Ferraron and Vogelstein. They believe that colorectal cancer develops from adenoma through a series of genetic events, including gene mutation and loss of tumor suppressor genes ${ }^{[4]}$. In addition to this type of precursor lesions, serrated polyps including proliferative polyps, sessile serrated adenomas/polyps and traditional serrated adenomas also have the probability of transforming into malignant tumors ${ }^{[5]}$. Lynch' syndrome caused by germline mutations in the mismatch repair gene (MMR) is the most common genetic form of colorectal cancer. In addition, there are about 12 cases of sporadic colorectal cancer with microsatellite instability (MSI) ${ }^{[6]}$. In many studies and reports, there is a metaanalysis on whether there is MSI in adenomas. It is found that MSI exists in varying degrees in colorectal adenomas related to Lynch syndrome or in primary adenomas ${ }^{[7]}$. Therefore, we will discuss MSI, colorectal adenomas, the MSI of Lynch syndrome and sporadic colorectal lesions, and the impact of MSI on the prognosis and treatment of colorectal cancer were elaborated and analyzed. 


\section{Microsatellite instability}

\subsection{Concept of microsatellite instability}

Microsatellite is a kind of repetitive DNA sequence with variable size and length of 1-6 nucleotides, so it is also called short tandem repeat, which widely exists in eukaryote genome. The function of DNA mismatch repair system is to prevent wrong insertion, deletion and base mismatch in the process of DNA replication and recombination. In the human genome, the repair function of MMR is highly conserved, which makes the genetic information present integrity. In human body, MSH2, MSH3, MSH6 and MLH1, MLH2, MLH3 are homologues of MutS and MutL as homodimers in prokaryotic MMR system, respectively. When a mismatch is detected in eukaryotic genomes, MMR system works through these dimers to recognize and repair the mismatch genes. If DNA mismatch repair genes ( $h M S H 2, h M L H 1$, hMSH3, hMLH3, hMSH6, hPMS1, hPMS2) have undergone mutation inactivation, microsatellite repeat sequence changes, that is, MSI may be resulted ${ }^{[8]}$. MSI can lead to the loss of accuracy of DNA replication and DNA repair after replication. Compared with cells with stable MMR system, the mutation frequency will increase by 100 to 700 times. MSI has been found in colorectal cancer, gastric cancer, endometrial cancer and other cancers, in which MSI colorectal tumors appear in the form of sporadic cancer or Lynch syndrome ${ }^{[9]}$.

Most MSI cancers have high immunogenicity, and the insertion/deletion mutation in the proteincoding region caused by MMR deficiency is considered as its potential mechanism. These mutations result in a high load of novel FSP antigens, including multiple HLA class I and II epitopes, and induce a significant immune response in the host. The immunogenicity produced by the high mutation load formed by this new antigen is the main reason why most MSI tumor patients respond better to the treatment of immune checkpoint blockade ${ }^{[10]}$. However, there are still MSI tumor patients who have not shown the benefit from immune checkpoint blockade. Therefore, certain experts speculate that this may be related to tumor immune evasion, and the mechanism of tumor immune evasion is the most important. In addition to the common mutation of $\beta 2$-microglobulin (B2M), it has recently been found that the mutation of human leukocyte antigen class I transactivator nlrc5 is also a potential immune escape mechanism ${ }^{[11]}$. Monitoring the typical immune evasion phenomenon of MSI cancer can help us to further understand the mechanism of immunotherapy resistance, and effectively predict the biomarkers of treatment response in patients with MSI colorectal cancer so as to improve the prediction accuracy and reduce the cost.

\subsection{Microsatellite instability detection method}

At home and abroad, the MSI detection of sample genes is usually conducted through the MSI analysis of fluorescent multiplex polymerase chain reaction (PCR) and the MMR protein analysis by immunohistochemistry (IHC) methods. The mutation status of 5 standard sites (BAT25, BAT26) in colorectal cancer tissues was detected. The expression of DNA mismatch repair genes ( $M L H 1, M S H 2$, MSH6 and PMS2) in colorectal cancer tissues was detected by IHC. Comparing the consistency of the two methods, the coincidence rate of the two methods is more than $90 \%$. It shows that performance of PCR and IHC are highly consistent in detecting microsatellite status ${ }^{[12]}$. There is a Bethesda for MSI detection/NCI panel, including BAT25, BAT26, D2S123, D5S346 and D17S250. This kind of microsatellite panel has become the gold standard for MSI detection, which has been reported for more than 20 years. If $30 \%$ or more loci showed instability, and the tumor was classified as MSI - High (MSI-H). If $1 \%$ to $29 \%$ of the markers were unstable, the tumor was classified as MSI - low (MSI-L). If no microsatellite markers showed instability, the tumor was classified as microsatellite stable (MSS). The Bethesda/NCI panel mainly relies on PCR and capillary electrophoresis fragment analysis for MSI detection ${ }^{[13]}$. In recent years, studies have found that a method can simultaneously amplify six single nucleotide repeat markers (BAT25, BAT26, $B A T 40, N R 21, N R 22, N R 27)$ and a polymorphic dinucleotide marker (D3S1260) in a single reaction. It is 
reported that this panel has higher sensitivity, especially for MSH6 deficient tumors ${ }^{[14]}$. In addition, the next generation sequencing (NGS) detection method by comparing the repeat length distribution of microsatellites has been added to the list of detection tools in recent years. The biggest advantage of NGS with $97 \%$ consistency rate with the traditional detection method is that it can detect microsatellite signals at a large number of microsatellite sites at the same time, and the accuracy is superior. The number of these microsatellite sites can range from several to hundreds of thousands ${ }^{[15]}$. Although the NGS method is still in its infancy, its prospect is worth looking forward to.

\section{Microsatellite instability analysis of colorectal cancer and polyps associated with Lynch syndrome}

\subsection{Lynch syndrome}

Lynch syndrome is a specific hereditary cancer syndrome, also known as hereditary nonpolyposis colorectal cancer (HNPCC). Lynch syndrome is usually associated with germline mutations. The susceptibility of this cancer is closely related to four key MMR genes (MLH1, MSH2, MSH6 and PMS2). Lynch syndrome may significantly increase the incidence of colorectal cancer, endometrial cancer, ovarian cancer, urothelial cancer, gastric cancer and other cancers ${ }^{[16]}$, and different MMR gene mutations may lead to different incidence of different cancers. For example, a 2020 study found that pathogenic $M L H 1$ and $M S H 2$ mutations lead to high exo-dominant cancer syndrome, and have similar colorectal cancer, endometrial cancer and other cancers. Among the carriers of MSH6 pathogenic mutation, the incidence of colorectal cancer was lower, but the risk of endometrial cancer was higher ${ }^{[17]}$. This suggests that we should develop different Lynch syndrome screening criteria for different MMR gene mutations. In families with known germline mutations, adenoma cancer sequence appeared more quickly, with an estimated residence time of 35 months, while sporadic cancer was about 10 years ${ }^{[18]}$, indicating the rapid progress of Lynch syndrome patients. Therefore, it is particularly important to identify Lynch syndrome patients in the early stage. It is best to accurately identify and diagnose the colorectal adenoma stage related to Lynch syndrome. Traditionally, Amsterdam II or revised Bethesda clinical standards are used, but it has been proven that such standards alone are insufficient in clinical practice ${ }^{[19]}$. Therefore, we can consider appropriately expanding the screening scope of Lynch syndrome. As Lynch syndrome can increase the prevalence and lifetime risk of various cancers, timely diagnosis of Lynch syndrome is very important.

\subsection{Lynch syndrome-related colorectal adenomas and polyps}

For Lynch syndrome-related colorectal adenomas and polyps, a series of studies have been carried out in many countries. For example, according to Masahiro Tanaka, 134 cases of colorectal adenomas in patients with Lynch syndrome confirmed by gene were detected by IHC. It was found that more than $80 \%$ of the samples had MMR defects ${ }^{[20]}$. These results indicate that most Lynch syndrome-associated adenomas have potential germline gene mutations, which usually correspond to MMR protein defects. Yurgelun MB et al. also detected microsatellite instability in 34 cases of colorectal polyps with MMR gene mutation from the reproductive system, including proliferative polyps, sessile serrated polyps and adenomatous polyps.One is MSI detection of BAT-25, NR-21, BAT-26, NR-24 and NR27, while another is to detect the expression of MLH1, MSH2, MSH6 and PMS2 by IHC. The results showed that nearly half of the polyps detected by PCR had MSI, 50\% of the adenomatous polyps showed abnormal IHC results, the other two kinds of polyps had low abnormal ratio, and there were larger $(>8 \mathrm{~mm})$ adenomatous polyps were more likely to have abnormal IHC results ${ }^{[21]}$. Although the total sample size of this experiment is not large, it suggests that MSI exists in adenomatous polyps of patients with family history or MMR protein expression loss in tumor (mmr-d). In other similar studies, the proportion of MSI instability in adenomatous polyps is higher, even as high as $70 \%$. These studies have certain clinical significance for the early detection of Lynch syndromerelated colorectal cancer in patients. A study found that MMR-deficient crypt lesions are a potential 
precursor of colorectal cancer in Lynch syndrome patients. Most of these lesions lack adenoma morphology, mainly single recessive or less recessive, which may be the earliest lesions in the development of Lynch syndrome-related colorectal tumors ${ }^{[22]}$. Therefore, some people think that MMR deficiency may have occurred before adenoma formation in Lynch syndrome-related colorectal tumorigenesis ${ }^{[23]}$. Some even speculate that this study suggests that colorectal cancer in Lynch syndrome may not necessarily go through the traditional adenoma stage ${ }^{[24]}$, so more experiments are needed to further investigate the mechanism of Lynch syndrome.

In addition, to optimize and improve the screening strategy of Lynch syndrome, we can optimize the selection of polyp characteristics or patient's age, family history, etc., for example, we can focus on young adenoma patients with family history of cancer, or young adenoma patients with high-risk characteristics, such as large volume, high atypical hyperplasia and malignant components, so that the evaluation of Lynch syndrome may be more reasonable ${ }^{[25]}$.

\subsection{Characteristics of Lynch syndrome-related colorectal cancer}

According to a number of studies, most Lynch syndrome-related MSI colorectal cancers are located in the proximal colon, with poor differentiation, tend to be mucinous, and are rich in infiltrating lymphocytes, as well as distant metastasis is rare ${ }^{[26]}$. Although the average age of Lynch syndrome patients diagnosed with colorectal cancer varies from country to country, the average age of Lynch syndrome patients is between 45 and 60 years old, which is slightly younger than that of sporadic cases (about 69 years old) ${ }^{[27]}$. Recently, other studies have found that routine diagnosis of colorectal cancer with MMR deficiency (dMMR) can effectively identify Lynch syndrome before 65 years old. However, in colorectal cancer patients aged 65 to 70 years old, due to the low prevalence of Lynch syndrome, routine detection of MMR is less effective in screening the disease in this population ${ }^{[28]}$.

We can see that the identification of MSI in pathological specimens is of great significance, not only because MSI molecular detection is an important means of screening ls, but also because it helps distinguish between microsatellite stable colorectal cancer and microsatellite unstable colorectal cancer, which is very valuable for prognosis estimation and individualized treatment.

\section{MSI of sporadic colorectal adenoma, polyp and carcinoma}

\subsection{Sporadic colorectal cancer}

Although genetic susceptibility plays a key role in some cases of colorectal adenoma and cancer, most colorectal diseases are non-hereditary sporadic lesions related to eating habits and obesity ${ }^{[29]}$. In terms of MSI of sporadic colorectal cancer, about $15 \%$ of sporadic colorectal cancer patients had MSI. The common gene change is MLH1 gene 5. Hypermethylation occurs in the CPG region, which will change the function of the promoter and adversely affect the normal transcription and expression [30]. In addition to the investigation of family genetic history, foreign studies have found that the incidence of BRAF mutation in sporadic colorectal cancer with MMR deficiency is high, and BRAF mutation is still a prognostic factor for MSI-H cancer. Since BRAF mutation almost does not occur with Lynch syndrome, we can further detect BRAF V600E mutation and MLH1 promoter hypermethylation so as to identify sporadic tumor or cancer caused by Lynch syndrome ${ }^{[31,32]}$. According to a recent study in China, in the MLH1-deficient colorectal cancer of Chinese population, although BRAF pre-screening for Lynch syndrome is highly sensitive, BRAF mutation is very rare. Therefore, BRAF detection and MLH1 promoter methylation detection will reduce the effectiveness of excluding Lynch syndrome in Chinese colorectal cancer patients ${ }^{[33]}$.

In the recent study on MSI of sporadic colorectal cancer, it was found that most of the patients with right colon cancer $(78.3 \%)$ were MSI-H, while most of the patients with left colon and rectum were MSS/MSI-L (78.6\% and 74.3\% MSS/MSI-L, respectively) ${ }^{[34]}$, which also showed that MSI-H colorectal 
cancer was mainly located in the right colon, which was consistent with the recent research results ${ }^{[35]}$. Sporadic colorectal cancer with dMMR is more likely to occur in women and older patients at the time of diagnosis than colorectal cancer associated with Lynch syndrome ${ }^{[36]}$. Sporadic MSI cancer is more common in poorly differentiated medullary carcinoma, and the positive expression rate of programmed death ligand 1 (PD-L1) is also higher ${ }^{[37]}$. This suggests that we may need to adopt different immunotherapy methods for sporadic MSI cancer and Lynch syndrome-related colorectal cancer, so as to achieve better therapeutic effect.

\subsection{Sporadic colorectal adenomas and polyps}

In addition to the well-known adenoma cancer sequence, there is another carcinogenic pathway of colorectal cancer through serrated polyps ${ }^{[38]}$. Therefore, certain experts thought that when studying the MSI of sporadic colorectal cancer, the adenoma and polyp and other precursor lesions should also be taken into account. In a recent study in Puerto Rico, after excluding patients with inflammatory bowel disease or family history of inflammatory bowel disease, IHC method was used to study the deletion of mismatch repair proteins in sporadic colon cancer and potentially large malignant adenomatous polyps. It was found that the deletion of mismatch repair proteins such as MSH1 existed not only in colorectal cancer, but also in primary colorectal cancer. There is a similar situation in primary adenoma ${ }^{[39]}$. Since the sample size of this study is relatively limited, it cannot be extended to all patients. However, it may suggest that we can trace the MSI of sporadic colorectal cancer patients to the early stage of adenoma and polyp. In fact, as early as 2010 or even earlier, some scholars believed that there was a close relationship between serrated polyps and sporadic colorectal cancer. Goel et al. have put forward that serrated polyps are the precursor of sporadic tumors with MSI ${ }^{[40]}$. There are also data showing that serrated polyps/adenomas are precursors of colorectal cancer caused by MSI and may rapidly develop into malignant tumors ${ }^{[41]}$. This suggests that in sporadic colorectal diseases, the serrated polyps of MSI are likely to develop into cancer in the later stage. It is suggested that surgeons should pay more attention to the evaluation and resection of serrated adenomas of colon because they may be one of the markers for the development of MSI sporadic cancer in the future.

\section{Prognostic and therapeutic value of MSI in colorectal cancer}

According to several reports on the survival rate of colorectal cancer, the risk of death and recurrence in patients with stage II MSI is lower than that in patients with MSS. However, some studies have found that in patients with stage III colon cancer, the survival rate of patients with MSI is worse than that of patients with MSS, and even the risk of invading nerves and lymphatic vessels is increased. For stage IV colorectal cancer with MSI, it has been reported that the prognosis of $\mathrm{H}$ depends on the mode of metastasis. For example, patients with peritoneal metastasis and MSI often have a good prognosis ${ }^{[42-44]}$. This shows that the prognosis of MSI colorectal cancer may be different with different tumor stages. At present, MSI testing is recommended for all patients with stage II colorectal cancer, but the sensitivity of colorectal cancer patients with MSI to adjuvant chemotherapy based on 5-FU is still controversial ${ }^{[45,46]}$. Some people even think that it is unwise to use MSI testing results to evaluate whether patients should receive adjuvant chemotherapy ${ }^{[47]}$. However, due to its highly up-regulated expression on various immune checkpoints, programmed cell death protein-1 (PD-1) and cytotoxic T lymphocyte associated protein-4 are gradually considered in the clinical setting. Among these therapeutic targets, CTLA-4 and PD-1 were found to be the most reliable targets. Pembrolizumab, an anti-PD-1 monoclonal antibody, can be used as one of the firstline treatment drugs for MSI-H/dMMR colorectal cancer, and it can also reduce the related adverse events [48-50]. MSI is a biomarker that responds to immune checkpoint inhibitors (ICPI). The response rate of colorectal cancer to immunosuppressive agents is variable. Responders have a higher burden of new antigens and a better response to immunotherapy than non-responders. This indicates that other predictive 
biomarkers are needed. Recent studies have found that the tumor mutation burden (TMB) may be MSI-H. TMB is an important biomarker in colorectal cancer, which is independent of MSI and PD-1 ligand PD-L1. When TMB is high, it will produce and present more peptide new antigens, and tumor cells are more likely to be recognized as allogeneic, thus initiating $\mathrm{T}$ cell activation and cytotoxic killing process, as well as increasing the response rate to immunotherapy ${ }^{[51]}$. It is found that MSI-H state is highly correlated with high TMB ${ }^{[52]}$.

\section{Summary and prospect}

Through a number of research results at home and abroad, we can draw the following conclusions: MSI is not only widely distributed in colorectal cancer, but also in adenomas and polyps closely related to colorectal cancer. According to the literature in recent years, it is found that serrated polyps are more likely to have MSI than other types of polyps. This suggests that we can start from this angle and try to gradually expand the scope of MSI screening. At present, there are few studies on MSI of various types of colorectal polyps compared with colorectal cancer, which indicates that the analysis of MSI of colorectal cancer precursor lesions is still not comprehensive, and thus, more investigations are still warranted in this field. MSI can not only be used to screen patients with LS, but also be beneficial to the individualized treatment of patients with various stages of colorectal cancer because of its unique pathological characteristics and high response rate to immunotherapy. Due to the lack of comprehensive and systematic research on the impact of MSI on the prognosis and treatment of colorectal cancer, it is necessary to conduct a more comprehensive study involving a large sample in the future.

\section{Funding}

Natural Science Foundation of Hebei Province: Beijing Tianjin Hebei basic research cooperation project (2019), Project No. 19JCZDJC65800(Z).

\section{Disclosure statement}

The authors declare that there is no conflict of interest.

\section{References}

[1] Sung H, Ferlay J, Siegel R L, et al., 2021, Global Cancer Statistics 2020: GLOBOCAN estimates of incidence and mortality worldwide for 36 cancers in 185 countries. CA: A Cancer Journal for Clinicians, 71(3): 209-249.

[2] Siegel RL, Miller KD, Jemal A, 2020, Cancer Statistics. CA: A Cancer Journal for Clinicians, 70(1).

[3] Araghi M, Soerjomataram I, Bardot A, et al., 2019, Changes in Colorectal Cancer Incidence in Seven High-Income Countries: A Population-Based Study. Lancet Gastroenterol Hepatol. 4: 511-518.

[4] Fearon ER, Vogelstein B. 1990, A Genetic Model for Colorectal Tumorigenesis. Cell. 61: 759-767.

[5] Okamoto K, Kitamura S, Kimura T, et al., 2016, Clinicopathological Characteristics of Serrated Polyps as Precursors to Colorectal Cancer: Current Status and Management. Journal of Gastroenterology \& Hepatology, 32(2): 358-367.

[6] Yuan L, Chi Y, Chen W, et al., 2015, Immunohistochemistry and Microsatellite Instability Analysis in Molecular Subtyping of Colorectal Carcinoma Based on Mismatch Repair Competency. Int J Clin Exp Med, 8(11): 20988-21000.

[7] Dabir PD, Bruggeling CE, Post RSVD, et al., 2019, Microsatellite Instability Screening in Colorectal 
Adenomas to Detect Lynch Syndrome Patients? A Systematic Review and Meta-analysis. European Journal of Human Genetics, 28: 277-286.

[8] Nojadeh JN, Sharif SB, Sakhinia E. 2018, Microsatellite Instability in Colorectal Cancer. EXCLI Journal, 17: 159-168.

[9] Angelika C, Dariusz W, Andrzej W, et al., 2017, Clinical Significance and Prognostic Relevance of Microsatellite Instability in Sporadic Colorectal Cancer Patients. International Journal of Molecular Sciences, 18(1): 107.

[10] Kloor M, von Knebel Doeberitz M, 2018, The Immune Biology of Microsatellite Unstable Cancer. Trends Cancer, 2(3): 121-133.

[11] Ozcan M, Janikovits J, von Knebel Doeberitz M, et al., 2018, Complex Pattern of Immune Evasion in MSI Colorectal Cancer. Oncoimmunology, 7(7): e1445453.

[12] Chen M, Chen Q, Zhou S, et al., 2018, Comparison of Microsatellite Status Detection Methods for Colorectal Cancer. Modern Oncology Medicine, 26(03): 0396-0400. (in Chinese)

[13] Baudrin LG, Jean-François D, How-Kit A, 2018, Molecular and Computational Methods for the Detection of Microsatellite Instability in Cancer. Frontiers in Oncology, 8: 621.

[14] Pagin A, Zerimech F, Leclerc J, et al., 2013, Evaluation of a New Panel of Six Mononucleotide Repeat Markers for the Detection of DNA Mismatch Repair-Deficient Tumours. British Journal of Cancer, 108(10): 2079-2087.

[15] Yamamoto G, Takenoya T, Takahashi A, et al., 2021, Quantitative Evaluation of MSI Testing Using NGS Detects the Imperceptible Microsatellite Changed Caused by MSH6 Deficiency. Familial Cancer, 20(2): 137-143.

[16] Win AK, Young JP, Lindor NM, 2012, Colorectal and Other Cancer Risks for Carriers and Noncarriers from Families with a DNA Mismatch Repair Gene Mutation: A Prospective Cohort Study. J Clin Oncol, 30: $958-964$.

[17] Dominguez-Valentin M, Sampson JR, Seppälä TT, et al., 2020, Cancer Risks by Gene, Age, and Gender in 6350 Carriers of Pathogenic Mismatch Repair Variants: Findings from the Prospective Lynch Syndrome Database. Genetics in Medicine, 22(1): 15-25.

[18] Edelstein DL, Axilbund JE, Baxter M, et al., 2011, Rapid Development of Colorectal Neoplasia in Patients with Lynch Syndrome. Clin Gastroenterol Hepatol, 9: 340-343.

[19] Goshayeshi L, Khooiee A, Ghaffarzadegan K, et al. 2017, Screening for Lynch Syndrome in Cases with Colorectal Carcinoma from Mashhad. Archives of Iranian Medicine, 20(6): 332-337.

[20] Tanaka M, Nakajima T, Sugano K, 2016, Mismatch Repair Deficiency in Lynch Syndrome-Associated Colorectal Adenomas is More Prevalent in Older Patients. Histopathology, 69: 322-328.

[21] Yurgelun MB, Goel A, Hornick JL, et al., 2012, Microsatellite Instability and DNA Mismatch Repair Protein Deficiency in Lynch Syndrome Colorectal Polyps. Cancer Prev Res (Phila), 5(4): 574-82.

[22] Kloor M, Huth C, Voigt AY, et al., 2012, Prevalence of Mismatch Repair - Deficient Crypt Foci in Lynch Syndrome: A Pathological Study. Lancet Oncol, 13: 598 - 606.

[23] Sekine S, Mori T, Ogawa R, et al., 2017, Mismatch Repair Deficiency Commonly Precedes Adenoma Formation in Lynch Syndrome-Associated Colorectal Tumorigenesis. Modern Pathology, 30(8): 11441151.

[24] Boland PM, Yurgelun MB, Boland CR, 2018, Recent Progress in Lynch Syndrome and Other Familial Colorectal Cancer Syndromes. CA: A Cancer Journal for Clinicians, 68(3): 217-231. 
[25] Zhu FC, Pan D, Zhang H, et al., 2019, Single-Center Study of Lynch Syndrome Screening in Colorectal Polyps. Hereditary Cancer in Clinical Practice, 17(1): 1-5.

[26] Chang Li, Chang M, Chang HM, et al., 2017, Expending Role of Microsatellite Instability in Diagnosis and Treatment of Colorectal Cancers. Journal of Gastrointestinal Cancer, 48(4): 305-313.

[27] Sinicrope FA, 2018, Lynch Syndrome-Associated Colorectal Cancer. N Engl J Med, 379: 764-773.

[28] Vos JR, Fakkert IE, Spruijt L, et al., 2020, Evaluation of Yield and Experiences of Age - Related Molecular Investigation for Heritable and Nonheritable Causes of Mismatch Repair Deficient Colorectal Cancer to Identify Lynch Syndrome. International Journal of Cancer, 147(8): 2150-2158.

[29] Gong Q, Zhang HH, Sun SB, et al., 2018, Mismatch Repair-Deficient Status Associates with Favorable Prognosis of Eastern Chinese Population with Sporadic Colorectal Cancer. Oncology Letters, 15(5): 7007-7013.

[30] Gatalica Z, Vranic S, Xiu J, et al., 2016, High Microsatellite Instability (MSI-H) Colorectal Carcinoma: A Brief Review of Predictive Biomarkers in the Era of Personalized Medicine. Familial Cancer, 15(3): 405-412.

[31] Lochhead P, Kuchiba A, Imamura Y, et al., 2013, Microsatellite Instability and BRAF Mutation Testing in Colorectal Cancer Prognostication. J Natl Cancer Inst, 105: 1151-1166.

[32] Loughrey MB, Waring PM, Tan A, et al., 2007, Incorporation of Somatic BRAF Mutation Testing into an Algorithm for the Investigation of Hereditary Non-Polyposis Colorectal Cancer. Familial Cancer, 6(3): 301-310.

[33] Xiao B, Luo J, Xie E, et al., 2020, Comparisons of Screening Strategies for Identifying Lynch Syndrome among Patients with MLH1-Deficient Colorectal Cancer. European Journal of Human Genetics, 28(11): 1555-1562.

[34] Atef N, Alieldin N, Sherif G, et al., 2020, Microsatellite Instability and Life Style Factors in Sporadic Colorectal Cancer. Asian Pacific Journal of Cancer Prevention: APJCP, 21(5): 1471.

[35] Bai H, Wang R, Cheng W, et al., 2020, Evaluation of Concordance between Deficient Mismatch Repair and Microsatellite Instability Testing and Their Association with Clinicopathological Features in Colorectal Cancer. Cancer Management and Research, 12: 2863.

[36] Sinicrope FA, Shi Q, Allegra CJ, et al., 2017, Association of DNA Mismatch Repair and Mutations in BRAF and KRAS with Survival after Recurrence in Stage III Colon Cancers: A Secondary Analysis of 2 Randomized Clinical Trials. JAMA Oncol, 3: 472-80.

[37] Yamada R, Yamaguchi T, Iijima T, et al., 2018, Differences in Histological Features and PD-L1 Expression between Sporadic Microsatellite Instability and Lynch-Syndrome-Associated Disease in Japanese Patients with Colorectal Cancer. International Journal of Clinical Oncology, 23(3): 504-513.

[38] Okamoto K, Kitamura S, Kimura T, et al., 2017, Clinicopathological Characteristics of Serrated Polyps as Precursors to Colorectal Cancer: Current Status and Management. Journal of Gastroenterology \& Hepatology, 32(2): 358-367.

[39] Reveron D, Lopez C, Gutierrez S, et al., 2018, Frequency of Mismatch Repair Protein Deficiency in a Puerto Rican Population with Colonic Adenoma and Adenocarcinoma. Cancer Genom Proteom. 15: 265-71.

[40] Boland CR, Goel A. 2010, Microsatellite Instability in Colorectal Cancer. Gastroenterology, 138(6):2073-2087.e3.

[41] Łukasz S, Janiczek M, Popiel A, et al., 2015, Serrated Polyps and Their Alternative Pathway to the Colorectal Cancer: A Systematic Review. Gastroenterology Research and Practice, 2015: 573814. 
[42] Petrelli F, Ghidini M, Cabiddu M, et al., 2019, Microsatellite Instability and Survival in Stage II Colorectal Cancer: A Systematic Review and Meta-analysis. Anticancer Research, 39(12): 6431-6441.

[43] Mohan HM, Ryan E, Balasubramanian I, et al., 2016, Microsatellite Instability is Associated with Reduced Disease Specific Survival in Stage III Colon Cancer. Eur J Surg Oncol, 42(11): 1680-1686.

[44] Fujiyoshi K, Yamamoto G, Takenoya T, et al., 2017, Metastatic Pattern of Stage IV Colorectal Cancer with High-Frequency Microsatellite Instability as a Prognostic Factor. Anticancer Research, 37(1): 239-248.

[45] Wood DE, 2015, National Comprehensive Cancer Network (NCCN) Clinical Practice Guidelines for Lung Cancer Screening. Thoracic Surgery Clinics, 25(2): 185-197.

[46] Webber EM, Kauffman TL, Elizabeth O, et al., 2015, Systematic Review of the Predictive Effect of MSI Status in Colorectal Cancer Patients Undergoing 5FU-Based Chemotherapy. BMC Cancer, 15: 156.

[47] Thomas ML, Hewett PJ, Ruszkiewicz AR, et al., 2015, Clinicopathological Predictors of Benefit from Adjuvant Chemotherapy for Stage C Colorectal Cancer: Microsatellite Unstable Cases Benefit. Asia Pacific Journal of Clinical Oncology, 11(4): 343-351.

[48] Losa NL, Cruise M, Tam A, et al., 2015, The Vigorous Immune Microenvironment of Microsatellite Instable Colon Cancer is Balanced by Multiple Counter-Inhibitory Checkpoints. Cancer Discov, 5(1): 43-51.

[49] Rotte A, 2019, Combination of CTLA-4 and PD-1 Blockers for Treatment of Cancer. Journal of Experimental, Clinical Cancer Research, 38(1): 255.

[50] Andre T, Shiu KK, Kim TW, et al., 2020, Pembrolizumab versus Chemotherapy for Microsatellite Instability-High/Mismatch Repair Deficient Metastatic Colorectal Cancer: The Phase 3 KEYNOTE177 Study.

[51] Schrock AB, Ouyang C, Sandhu J, et al., 2019, Tumor Mutational Burden is Predictive of Response to Immune Checkpoint Inhibitors in MSI-High Metastatic Colorectal Cancer. Annals of Oncology, 30(7): 1096-1103.

[52] Fabrizio DA, George Jr TJ, Dunne RF, et al., 2018, Beyond Microsatellite Testing: Assessment of Tumor Mutational Burden Identifies Subsets of Colorectal Cancer Who May Respond to Immune Checkpoint Inhibition. Journal of Gastrointestinal Oncology, 9(4): 610. 